\title{
FUNÇÃO QUADRÁTICA E ÁREA MÁXIMA DE RETÂNGULO EM LIVROS DIDÁTICOS DO ENSINO MÉDIO
}

\section{QUADRATIC FUNCTION AND MAXIMUM AREA OF RECTANGLES IN HIGH SCHOOL TEACHING BOOKS}

\author{
Jeandson Moura dos Santos ${ }^{1}$; Elisângela Bastos de Mélo Espíndola²
}

\begin{abstract}
RESUMO
Apresentamos o recorte de um trabalho de conclusão de curso, desenvolvido na Licenciatura em Matemática (UFRPE), cujo objetivo é analisar a praxeologia matemática de situações-problema envolvendo Função Quadrática e Área Máxima de Retângulo apresentadas em livros didáticos do Ensino Médio. Como referencial teórico nos norteamos pela Teoria Antropológica do Didático, desenvolvida no seio da Didática da Matemática francesa. Metodologicamente, esta pesquisa tem uma abordagem qualitativa e é do tipo documental. Nos procedimentos de coleta e análise de dados, consultamos oito livros didáticos (LD) do $1^{\circ}$ ano do Ensino Médio, de coleções aprovadas no Programa Nacional do Livro Didático (2018). Especificamente, no capítulo sobre Função Quadrática buscamos analisar o tópico de atividades para resposta do aluno, no tópico específico sobre Valor Máximo e Valor Mínimo da Função Quadrática. Detemo-nos em realizar a referida análise no bloco prático-técnico da organização matemática em tela; ou seja, em torno dos tipos de técnicas e de tarefas. Dentre os resultados, foram identificados três tipos de tarefas (T): T1. Calcular as medidas dos lados de um retângulo para que sua área seja máxima; T2. Calcular a medida da área máxima de um retângulo e T3. Comparar a área máxima de dois retângulos. Para cada uma dessas tarefas, discorremos sobre seus subtipos e técnicas apresentadas pelos autores dos livros didáticos. Percebemos que de oito LD analisados em seis deles abordaram os tipos de tarefa T1, T2 e T3. Isto é, constatamos que o LD4 e o LD5, não apresentaram situações-problema, propostas à resolução dos alunos, para estes tipos de tarefas. De modo geral identificamos quatro situações-problema relacionadas à T1, quatro relacionadas à T2 e apenas uma para T3. O LD1 e o LD3 foram aqueles que apresentaram a maior diversidade de tarefas.

Palavras-chave: Função Quadrática; Área Máxima de Retângulo; Livro Didático; Teoria Antropológica do Didático.
\end{abstract}

\footnotetext{
ABSTRACT

We present an excerpt from a course conclusion work, developed in the Mathematics Degree (UFRPE), whose objective is to identify the mathematical praxeology of problem situations

${ }^{1}$ Mestrando em Ensino das Ciências, Universidade Federal Rural de Pernambuco (UFRPE). Endereço para correspondência: Rua da Serraria, 20, Cidade de Deus - Bonança, Moreno, Pernambuco, Brasil, CEP: 54800-000. E-mail: jeandsonmoura@ hotmail.com.

ORCID iD: https://orcid.org/0000-0003-0374-4972.

2 Doutora em Educação, Universidade Federal de Pernambuco (UFPE). Professora adjunta do Departamento de Educação (UFRPE), Recife, Pernambuco, Brasil. Endereço para correspondência: Rua Dom Manuel de Medeiros, S/N, Departamento de Educação, Dois Irmãos, Recife, Pernambuco, Brasil, CEP: 52171-900. E-mail: elisangela.melo@ufrpe.br.

(iD) ORCID iD: https://orcid.org/0000-0002-3769-0768.
} 
involving Quadratic Function and Maximum Area of Rectangle presented in high school textbooks. As a theoretical reference we are guided by the Anthropological Theory of Didactics, developed within the Didactics of French Mathematics. Methodologically, this research has a qualitative approach and is of the documentary type. In the procedures for data collection and analysis, we consulted eight textbooks (LD) from the 1st year of high school, from collections approved in the National Textbook Program (2018). Specifically, in the chapter on Quadratic Function we seek to analyze the topic of activities for student response, in the specific topic about Maximum and Minimum Value of the Quadratic Function. We pause to carry out this analysis in the practical-technical block of the mathematical organization on screen; that is, around the types of techniques and tasks. Among the results, three types of tasks (T) were identified: T1. Calculate the measurements of the sides of a rectangle so that its area is maximum; T2. Calculate the measure of the maximum area of a rectangle and T3. Compare the maximum area of two rectangles. For each of these tasks, we discuss their subtypes and techniques presented by the textbook authors. We noticed that out of eight LD analyzed, only six of them addressed task types T1, T2 and T3. That is, we found that LD4 and LD5 did not present problem situations, proposed to the students' resolution, for these types of tasks. In general, we identified four problem situations related to T1, four related to T2 and only one for T3. LD1 and LD3 were those that presented the greatest diversity of tasks.

Keywords: Quadratic Function; Maximum Rectangle Area; Textbook; Anthropological Theory of Didactics. 


\section{Introdução}

Este trabalho teve por objetivo analisar a praxeologia matemática de situaçõesproblema envolvendo Função Quadrática e Área Máxima de Retângulo em livros didáticos (LD) do Ensino Médio. A escolha deste tema se ancora em pesquisas, a exemplo de Chaves (2016, p.6), que considera o estudo de Função Quadrática fundamental para:

O domínio do conhecimento matemático, pelas conexões que se estabelecem entre esta e outros estudos da própria Matemática, pela sua aplicação em situações-problema do cotidiano, assim como em diferentes domínios, tais como Física, Química, Biologia, Economia, Administração e Engenharia.

Em busca de atingir o objetivo da pesquisa, apresentamos algumas considerações sobre elementos da Teoria Antropológica do Didático (TAD) (CHEVALLARD, 1992) que utilizamos como suporte à análise da organização matemática dos LD. Neste sentido concordamos com Bittar (2017, p. 365-366), ao afirmar que:

Se queremos compreender algumas das razões de dificuldades de aprendizagem enfrentadas por alunos, o livro didático utilizado por eles é uma das fontes a serem consultadas. Não é a única, porém, como o LD é o principal material utilizado pelo professor no preparo de suas aulas, seu estudo permite, entre outros, certa aproximação com o que é ensinado pelo professor.

Chaachoua e Comiti (2010, p. 771) consideram que a análise do LD se tornou "uma entrada indispensável para compreender o funcionamento ou para caracterizar o estado do sistema em um dado instante". Desta forma, a TAD tem sido tomada como um aporte para análise de LD, tendo em vista a apreensão das relações institucionais em torno de um dado saber em jogo.

Os LD são "produtos de instituições transpositivas" (CHAACHOUA; COMITI, 2010, p. 772). Ou seja, os LD são considerados como o resultado de uma transposição didática dos textos de programas curriculares. Eles podem se diferenciar segundo as escolhas dos autores. No entanto, na maioria dos casos, eles são a tradução de uma diretiva institucional, que se exprime frequentemente pelas orientações curriculares, "segundo uma interpretação dos autores" (idem). Em virtude do exposto, para melhor compreensão dos elementos da TAD, refinamos a seguir algumas considerações sobre esta teoria e na sequência apresentamos os procedimentos metodológicos adotados para a análise dos LD. 


\section{Considerações sobre a Teoria Antropológica do Didático}

A TAD, elaborada por Chevallard (1992), é complementar aos estudos sobre a transposição didática do objeto matemático e se constitui como uma ferramenta que possibilita a análise das transformações que são realizadas nos objetos de saber a ensinar, no seio das instituições.

A TAD oferece as ferramentas que possibilitam desvelar as organizações matemáticas e didáticas usuais nestas instituições no trabalho com os objetos de ensino e aprendizagem. Nesse sentido, o conjunto composto por essas organizações (matemáticas e didáticas) é um patrimônio da instituição escolar, da instituição livro didático de matemática, da instituição professor, por exemplo. Araújo (2009, p. 20) afirma que a TAD "permite descrever e estudar as condições de existência dos objetos dos saberes nas instituições de ensino".

Segundo Chevallard (1992), para determinado tema de estudo deve-se considerar, em primeiro lugar, a realidade matemática que pode ser construída, a qual chama de Praxeologia Matemática ou Organização Matemática (OM); em segundo lugar, a maneira como essa realidade pode ser estudada, que será denominada Organização Didática (OD).

A noção de praxeologia matemática é instituída com base nos tipos de tarefas (T) a serem realizadas por meio da técnica $(\tau)$, que, por sua vez, é explicada e legitimada por elementos tecnológicos $(\theta)$, justificados e esclarecidos por uma teoria $(\Theta)$. A praxeologia $[\mathrm{T}, \tau, \theta, \Theta]$ formada por esses quatro componentes articula um bloco prático-técnico [T, $\tau$ ], designando o saber-fazer, que consiste a associação entre certo tipo de tarefa e uma determinada técnica, e um bloco tecnológico-teórico $[\theta, \Theta]$, designando o saber, resultado da articulação entre a tecnologia e a teoria (ARAÚJO, 2009).

No presente trabalho, vamos abordar a praxeologia matemática, em particular o bloco prático-técnico $[\mathrm{T}, \tau]$, designando o saber-fazer, que consiste na associação entre certo tipo de tarefa e uma determinada técnica.

\section{Metodologia}

A presente pesquisa se insere numa abordagem qualitativa. De acordo com Godoy (1995, p. 23): “A abordagem qualitativa, enquanto exercício de pesquisa, não se apresenta como uma proposta rigidamente estruturada, ela permite que a imaginação e a criatividade levem os investigadores a propor trabalhos que explorem novos enfoques". 
O estudo sobre os LD do Ensino Médio foi empreendido a partir de uma análise documental. Segundo Flick (2009, p. 234), neste tipo de pesquisa:

O pesquisador deve entender os documentos como "meios de comunicação", pois foram elaborados com algum propósito e para alguma finalidade, sendo inclusive destinado para que alguém tivesse acesso a eles. Assim, indica que é importante compreender quem o produziu, sua finalidade, para quem foi construído, a intencionalidade de sua elaboração.

O estudo em uma pesquisa qualitativa de caráter documental, de acordo com Chaves (2016, p.59), busca "dar respostas às atitudes ou procedimentos humanos subjacentes nas abordagens contextuais apresentadas nos documentos (livros didáticos de matemática)". No nosso caso, a análise documental foi realizada em oito LD de coleções aprovadas pelo Programa Nacional do Livro Didático do Ensino Médio (2018), a saber:

Quadro 1 - Coleções de livros didáticos (PNLD 2018).

\begin{tabular}{|c|l|}
\hline LD & \multicolumn{1}{c|}{ Coleções } \\
\hline LD1 & Matemática Ciência e Aplicações (IEZZI et al., 2017) \\
\hline LD2 & Matemática Interação e Tecnologia (BALESTRI, 2016) \\
\hline LD3 & \#Contato Matemática (SOUZA; GARCIA, 2016) \\
\hline LD4 & Quadrante Matemática (CHAVANTE; PRESTES, 2016) \\
\hline LD5 & Matemática Contexto \& Aplicações (DANTE, 2017) \\
\hline LD6 & Conexões com a Matemática (LEONARDO, 2016) \\
\hline LD7 & Matemática Ensino Médio (SMOLE; DINIZ, 2014) \\
\hline LD8 & Matemática Paiva (PAIVA, 2015) \\
\hline
\end{tabular}

Fonte: Elaborado pelos autores (2021).

Sobre os LD (Quadro 1), foram selecionados aqueles do $1^{\circ}$ ano do Ensino Médio por conter o capítulo referente à Função Quadrática. A análise dos capítulos sobre Função Quadrática ocorreu na parte dos LD do aluno: "Atividades propostas". Além disso consultamos o manual de orientações no LD do professor. Nesse momento buscamos:

\begin{abstract}
Analisar cada atividade identificando qual é a tarefa do aluno e qual é a técnica que se espera que ele utilize para a resolução da tarefa, tendo como apoio a(s) praxeologia(s) anteriormente identificada(s). Uma pergunta recorrente, e totalmente pertinente, é “como saber que essa é a técnica que o autor gostaria que fosse usada?" Para realizar tal inferência baseamo-nos no que está presente no Manual do Professor [...]. Por meio dessa análise procuramos elementos que permitam inferir sobre como os autores do LD desejariam que seus usuários resolvessem as atividades (BITTAR, 2017, p.373).
\end{abstract}

No Quadro 2, pode ser vista a distribuição de 13 situações-problema analisadas em cada LD do aluno em "Atividades propostas", no tópico específico: Valor Máximo ou Mínimo de uma Função Quadrática. 
Quadro 2 - Situações-problema analisadas por LD.

\begin{tabular}{|c|c|}
\hline LD & Quantidade \\
\hline LD1 & 4 \\
\hline LD2 & 1 \\
\hline LD3 & 3 \\
\hline LD4 & 0 \\
\hline LD5 & 0 \\
\hline LD6 & 2 \\
\hline LD7 & 1 \\
\hline LD8 & 2 \\
\hline
\end{tabular}

Fonte: Elaborado pelos autores (2021).

Para a análise dos tipos de tarefas e de técnicas, fomos selecionando cada uma delas e analisando-os a fim de organizar os dados em quadros descritivos com alguns exemplos. Ressaltamos que no processo de análise das técnicas, consultamos o manual do professor, a fim de identificar a técnica esperada pelos autores, ou seja, aquela a ser utilizada pelo aluno na resolução da tarefa.

\section{Análise dos resultados}

No Quadro 3, apresentamos os tipos de tarefas identificadas em situaçõesproblema, no tópico “Atividades propostas”, sobre Função Quadrática e Área Máxima de Retângulo em LD do Ensino Médio.

Quadro 3 -Tipos de tarefas sobre Função Quadrática e Área Máxima de um retângulo. T1. Calcular as medidas dos lados de um retângulo para que sua área seja máxima.

$\mathrm{T}_{1.1}$. Calcular as medidas dos lados de um retângulo para que sua área seja máxima, sendo dada a medida do seu perímetro.

$\mathrm{T}_{1.2 \text {. }}$ Calcular as medidas dos lados de um retângulo para que sua área seja máxima, sendo dada a medida do seu perímetro mais a medida do segmento de reta que subdivide esse retângulo em duas partes.

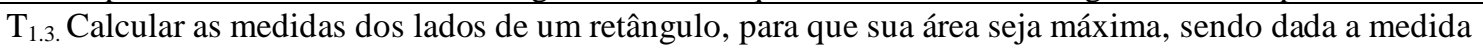
do comprimento dos dois lados menores, da medida do segmento de reta que subdivide esse retângulo em duas partes mais a medida de "um" dos lados maiores do retângulo.

$\mathrm{T}_{1.4}$. Calcular as medidas dos lados de um retângulo para que sua área seja máxima, sendo dada a medida do comprimento dos dois lados menores mais a medida de "um" dos lados maiores do retângulo, multiplicada por 4 .

T2. Calcular a medida da área máxima de um retângulo.

$\mathrm{T}_{2.1}$. Calcular a área máxima de um retângulo, sendo dada a medida do seu perímetro.

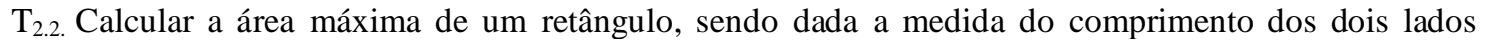
menores mais a medida de "um" dos lados maiores do retângulo.

T3. Comparar a área máxima de dois retângulos.

$\mathrm{T}_{3.1 \text {. }}$ Comparar a área máxima de um retângulo nos casos de $\mathrm{T}_{1.2}$ e de $\mathrm{T}_{1.3}$.

Fonte: Elaborado pelos autores (2021). 
Identificamos o subtipo de tarefa " $\mathrm{T}_{1.1}$ - Calcular as medidas dos lados de um retângulo para que a sua área seja máxima, sendo dada a medida do seu perímetro" no LD3 (primeira questão) e no LD7.

Figura 1 - Exemplos de $\mathrm{T}_{1.1}$ no LD3 e no LD7.

\begin{tabular}{|c|c|}
\hline Exemplo do LD3 & Exemplo do LD7 \\
\hline 45. Pedro pretende cercar uma região retangular em \\
sua chácara para criar galinhas. Para isso, ele \\
comprou 80 m de tela e pretende usá-la de \\
$\begin{array}{l}\text { modo a obter a maior área possível para o gali- } \\
\text { nheiro. Quais devem ser as medidas dos lados } \\
\text { desse galinheiro? Qual será a área máxima des- } \\
\text { se galinheiro? Os lados devem ter } 20 \mathrm{~m} ; 400 \mathrm{~m}^{2}\end{array}$ & 31. Dispomos de uma tela de arame com 28 metros de com- \\
Fonte: Souza e Garcia $(2016, \mathrm{p} .126)$ & ser as medidas dos lados do retângulo para que a área \\
\end{tabular}

Para $\mathrm{T}_{1.1}$ (Quadro 4), ambos os autores dos LD3 e LD7 propõem a técnica $\tau_{1.1}$.

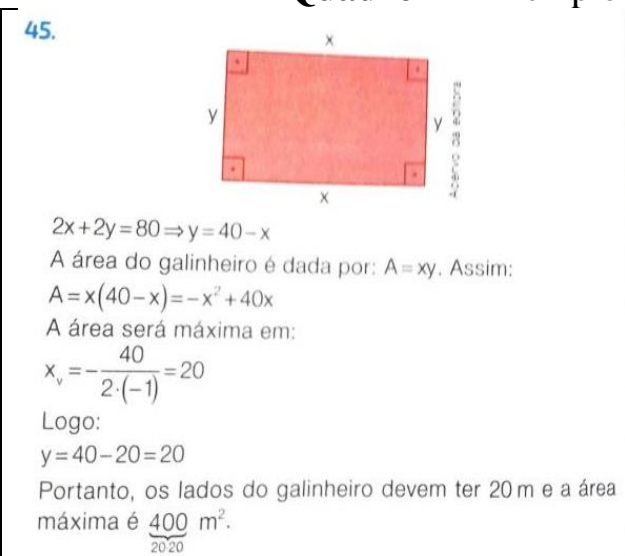

Fonte: Souza e Garcia (2016, p.126)

Quadro 4 - Exemplo de técnica relacionada a $\mathrm{T}_{1.1}$ no LD3.

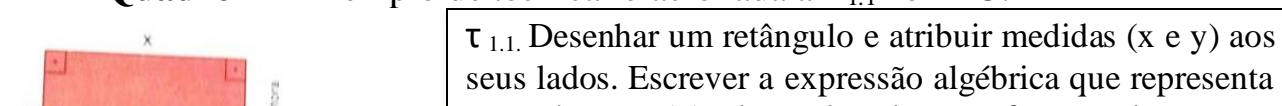
$\mathrm{o}$ perímetro $(\mathrm{P})$ do retângulo em função de $\mathrm{x}$ e $\mathrm{y}$ $(2 x+2 y=P)$. Simplificar a expressão algébrica. Expressar a medida y em função de $\mathrm{x}$. Escrever a expressão algébrica que representa a área do retângulo em função de $\mathrm{x}$ e y $(A=x y)$. Substituir a medida y (que foi expressa em função de $\mathrm{x}$ ) na expressão algébrica que representa a área do retângulo. Aplicar a propriedade distributiva para se obter a expressão algébrica na forma $\mathrm{A}(\mathrm{x})=\mathrm{ax}^{2}+\mathrm{bx}$. Determinar a medida da medida de $\mathrm{x}$ (para área máxima) a partir do cálculo das coordenadas do vértice da parábola, usando $\mathrm{x}_{\mathrm{v}}=-\frac{b}{2 a}$. Calcular a medida $\mathrm{y}$, sabendo a medida $\mathrm{x}$ (para área máxima) na expressão algébrica que
representa P. Indicar a medida de x ey.

Fonte: Elaborado pelos autores (2021).

O subtipo de tarefa " $\mathrm{T}_{1.2}$ - Calcular as medidas dos lados de um retângulo, sendo dada a medida do seu perímetro mais a medida do segmento de reta que subdivide esse retângulo em duas partes - foi identificado apenas no LD1, no “item b” (Quadro 5). 
Quadro 5 - Exemplo de $\mathrm{T}_{1.2 \mathrm{e}} \tau_{1.2}$ no LD1.

\begin{tabular}{|c|c|}
\hline $\mathbf{T}_{1.2}$ & Técnica e \\
\hline $\begin{array}{l}32 \text { Um fazendeiro possui } 150 \text { metros de um rolo de } \\
\text { tela para cercar um jardim retangular e um pomar, } \\
\text { aproveitando, como um dos lados, parte de um } \\
\text { muro, conforme indica a figura seguinte: } \\
\text { a) Para cercar com a tela a maior área possível, } \\
\text { quais devem ser os valores de } \mathbf{x} \text { e } \mathbf{y} \text { ? } \\
\text { b) Qual seria a resposta, caso não fosse possivel apro- } \\
\text { veitar a parte do muro indicada, sendo necessário } \\
\text { cercá-la com a tela? Nesse caso, em que percen- } \\
\text { tual ficaria reduzida a área máxima da superfície } \\
\text { limitada pelo jardim e pelo pomar reunidos? }\end{array}$ & 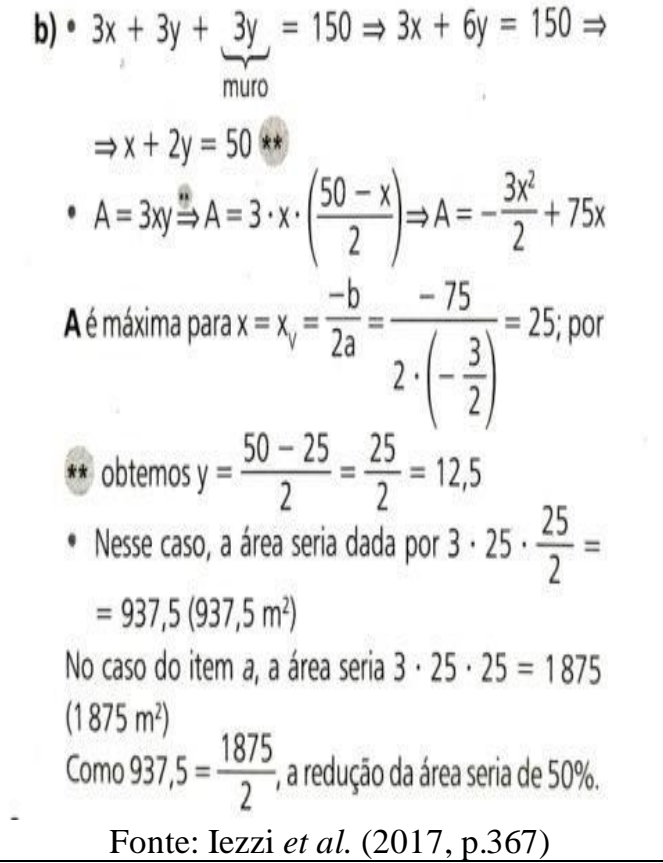 \\
\hline \multicolumn{2}{|c|}{$\begin{array}{l}\mathrm{t}_{1.2} \text {. Escrever a expressão algébrica que representa o perímetro }(\mathrm{P}) \text { do retângulo mais o segmento de reta } \\
\text { que o subdivide em duas partes, em função de } \mathrm{x} \text { e } \mathrm{y}(3 \mathrm{x}+3 \mathrm{y}+3 \mathrm{y}=150) \text {. Simplificar a expressão algébrica. } \\
\text { Expressar a medida y em função de } \mathrm{x} \text {. Escrever a expressão algébrica que representa a área do retângulo } \\
\text { em função de } \mathrm{x} \text { e y }(\mathrm{A}=3 \mathrm{xy} \text { ). Substituir a medida y (que foi expressa em função de } \mathrm{x} \text { ) na expressão } \\
\text { algébrica que representa a área do retângulo. Aplicar a propriedade distributiva para se obter a expressão } \\
\text { algébrica na forma } \mathrm{A}(\mathrm{x})=\mathrm{ax}{ }^{2}+\mathrm{bx} \text {. Determinar a medida de } \mathrm{x} \text { (para área máxima) a partir do cálculo } \\
\text { das coordenadas do vértice da parábola, usando } \mathrm{x}_{\mathrm{v}}=-\frac{b}{2 a} \text {. Calcular a medida y, sabendo a medida } \mathrm{x} \\
\text { (para área máxima) na expressão algébrica que representa } \mathrm{P} \text {. Indicar as medidas } \mathrm{x} \text { e y. }\end{array}$} \\
\hline
\end{tabular}

Fonte: Elaborado pelos autores (2021).

No LD1 (Quadro 5), no "item a", temos o subtipo de tarefa " $\mathrm{T}_{1.3}$. Calcular as medidas dos lados de um retângulo, para que sua área seja máxima, sendo dada a medida do comprimento dos dois lados menores, da medida do segmento de reta que subdivide esse retângulo em duas partes mais a medida de "um" dos lados maiores do retângulo. 
Quadro 6 - Exemplo de $\tau_{1.3}$ para resolver $T_{1.3}$ no LD1.

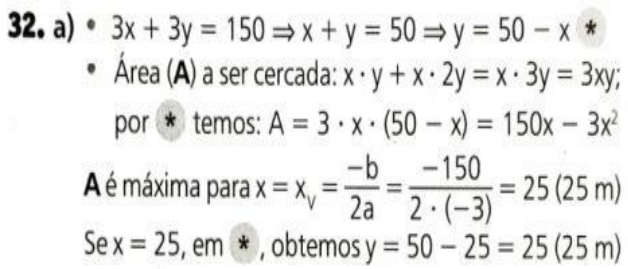

Fonte: Iezzi et al. (2017, p.367).

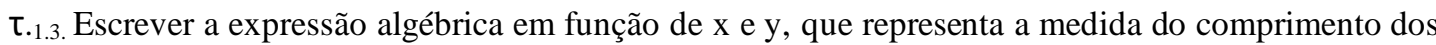
dois lados menores de um retângulo, do segmento de reta que o subdivide em duas partes mais a medida de um dos lados maiores do retângulo $(3 x+3 y=150)$. Simplificar a expressão algébrica. Escrever a expressão algébrica que representa a área dos dois retângulos em função de x e y $(A=x \cdot y+x .2 y)$. Substituir a medida y (em função de $\mathrm{x}$ ) na expressão algébrica que representa a área do retângulo. Aplicar a propriedade distributiva para se obter a expressão algébrica na forma $\mathrm{A}(\mathrm{x})=\mathrm{ax}{ }^{2}+\mathrm{bx}$. Determinar a medida de $\mathrm{x}$ (para área máxima) a partir do cálculo das coordenadas do vértice da parábola, usando $\mathrm{x}_{\mathrm{v}}$ $=-\frac{b}{2 a}$. Calcular a medida $\mathrm{y}$, sabendo a medida $\mathrm{x}$ (para área máxima) na expressão algébrica $\mathrm{y}=50-\mathrm{x}$. Indicar as medidas $\mathrm{x}$ e $\mathrm{y}$.

Fonte: Elaborado pelos autores (2021).

Quanto ao subtipo de tarefa " $\mathrm{T}_{3.1}$ - Comparar a área máxima de um retângulo nos casos de $T_{1.2}$ e de $T_{1.3}$, esse subtipo de tarefa foi identificado apenas no LD1, na segunda questão do item "b" (Quadro 5). Com o uso da seguinte técnica (Figura 2): " $\tau_{3.1}$. Aplicar $\tau_{1.3}$ e $\tau_{1.4}$. Comparar os resultados obtidos em $\tau_{1.3}$ e $\tau_{1.4}$. Determinar o percentual de redução da área máxima de uma situação para outra".

Figura 2 - Exemplo de $\tau_{3.1}$ para resolver $\mathrm{T}_{3.1}$ no LD1.

- Nesse caso, a área seria dada por $3 \cdot 25 \cdot \frac{25}{2}=$ $=937,5\left(937,5 \mathrm{~m}^{2}\right)$

No caso do item $a$, a área seria $3 \cdot 25 \cdot 25=1875$ $\left(1875 \mathrm{~m}^{2}\right)$ Como 937, $5=\frac{1875}{2}$, a redução da área seria de 50\%.

Fonte: Iezzi et al. (2017, p.367).

O subtipo de tarefa " $T_{1.4}$ - Calcular as medidas dos lados de um retângulo para que sua área seja máxima, sendo dada a medida do comprimento dos dois lados menores mais a medida de "um" dos lados maiores do retângulo, multiplicada por 4. - foi identificado no LD2 e no LD3. No Quadro 7, podemos ver um exemplo desse subtipo de tarefa, identificado no LD3. 
Quadro 7 - Exemplo de $T_{1.4}$ e $\tau_{1.4}$ no LD3.

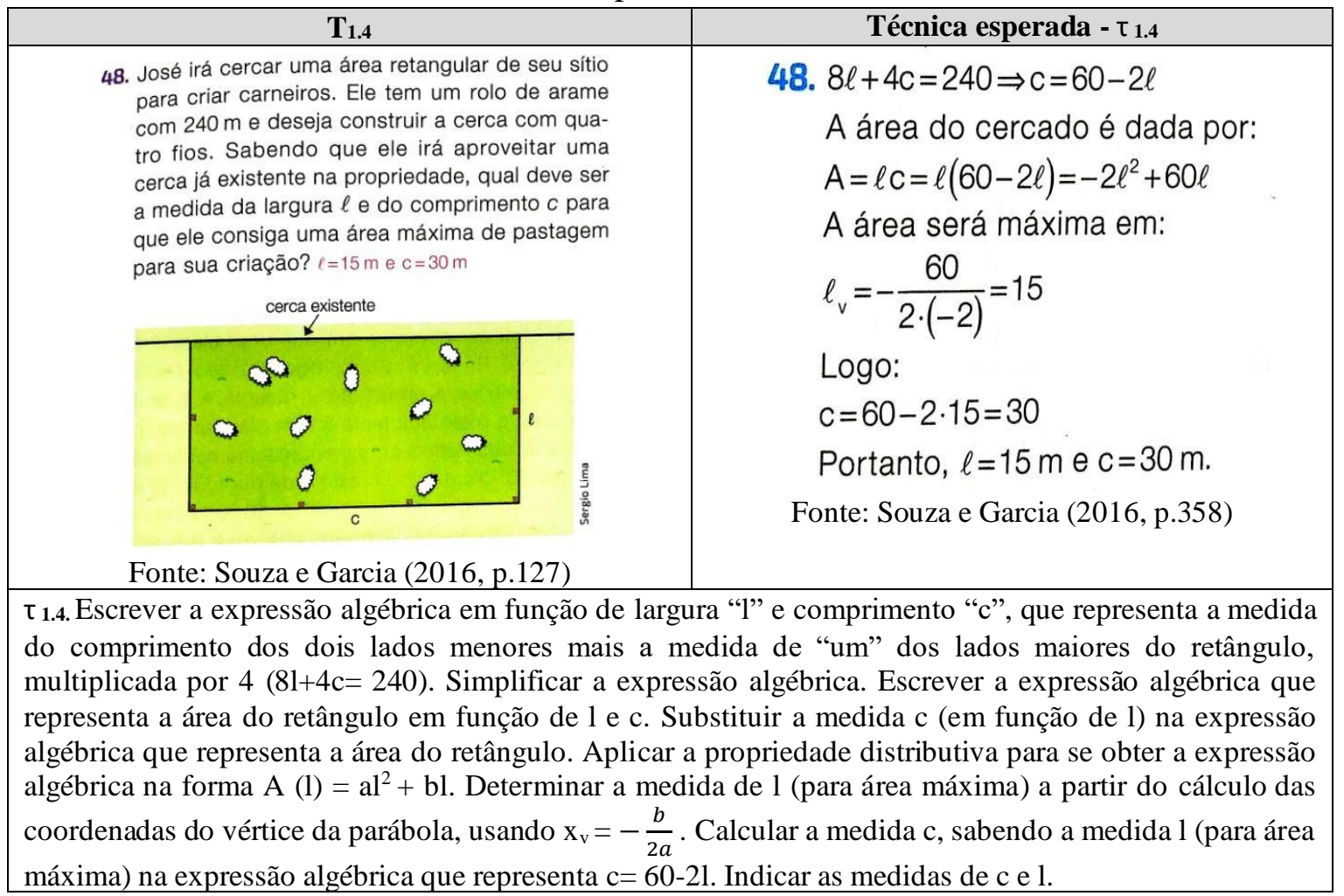

Fonte: Elaborado pelos autores (2021).

Quanto ao subtipo de tarefa "T $\mathrm{T}_{2.1}$. Calcular a área máxima de um retângulo, sendo dada a medida do seu perímetro", esse foi reconhecido no LD1, LD3, LD6 e LD8. No Quadro 8 podemos ver um exemplo de $\mathrm{T}_{2.1}$, identificado no LD1.

Quadro 8 - Exemplo de $\mathrm{T}_{2.1}$ e $\tau_{2.1}$ no LD1

\begin{tabular}{|c|c|}
\hline $\mathbf{T}_{2.1}$ & Técnica esperada - $\tau_{2.1}$ \\
\hline $\begin{array}{l}33 \text { Entre todos os retângulos de perímetro } 20 \text { cm, } \\
\text { determine aquele cuja área é máxima. Qual é essa } \\
\text { área? } \\
\text { Fonte: Iezzi et al. (2017, p. } 105)\end{array}$ & 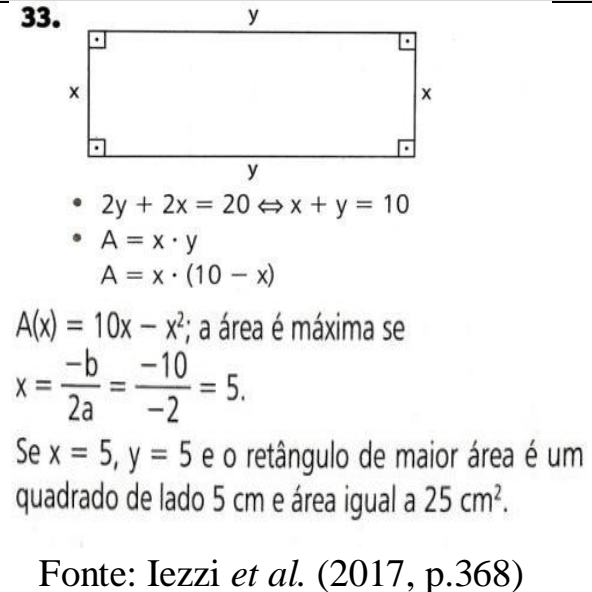 \\
\hline
\end{tabular}

Fonte: Elaborado pelos autores (2021). 
No exemplo a seguir, o subtipo de tarefa $\mathrm{T}_{2.1}$ foi proposto no LD8, no item c (Figura 3), com uma técnica diferente $\left(\tau_{2.2}\right)$ daquela apresentada no LD1. No Quadro 9, podemos ver que $\tau_{2.2}$ requer a construção do gráfico da Função Quadrática.

Figura 3 - Exemplos de $T_{2.1}$ no LD8

10) Considere todos os retângulos com $20 \mathrm{~cm}$ de perímetro.

a) Entre eles, qual é a área do retângulo com $8 \mathrm{~cm}$ de base? $16 \mathrm{~cm}^{2}$

b) Entre eles, indicando por $x$ a medida da base de um retângulo genérico, construa 0 gráfico da função $A(x)$ que expressa a área do retângulo, em centímetro quadrado, em função da medida $x$, em centímetro.

Ver' Suplemento com orientaçōes para o professor.

c) Qual é a área máxima que pode ter um desses retângulos? $25 \mathrm{~cm}^{2}$

Fonte: Paiva (2015, p. 188)

Quadro 9-Exemplo de $\tau_{2.2}$ no LD8 pata $\mathrm{T}_{2.1}$

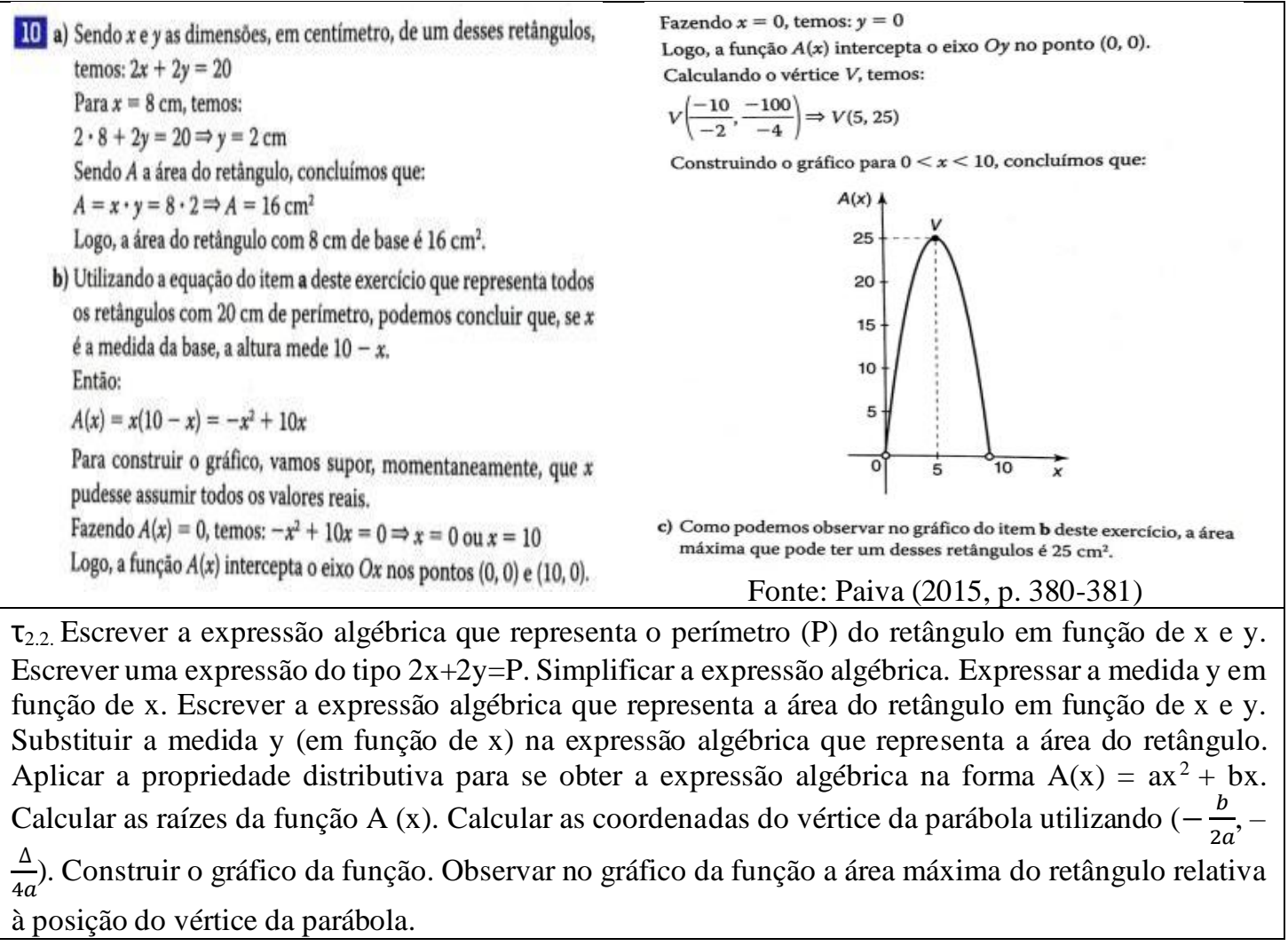

Fonte: Elaborado pelos autores (2021).

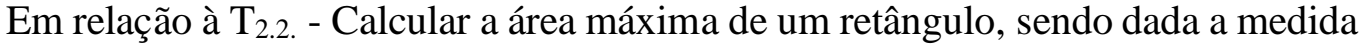
do comprimento dos dois lados menores mais a medida de "um" dos lados maiores do retângulo - esse subtipo de tarefa foi identificado no LD8. 
Quadro 10 - Exemplo de T2.2 e $\tau 2.3$ no LD8

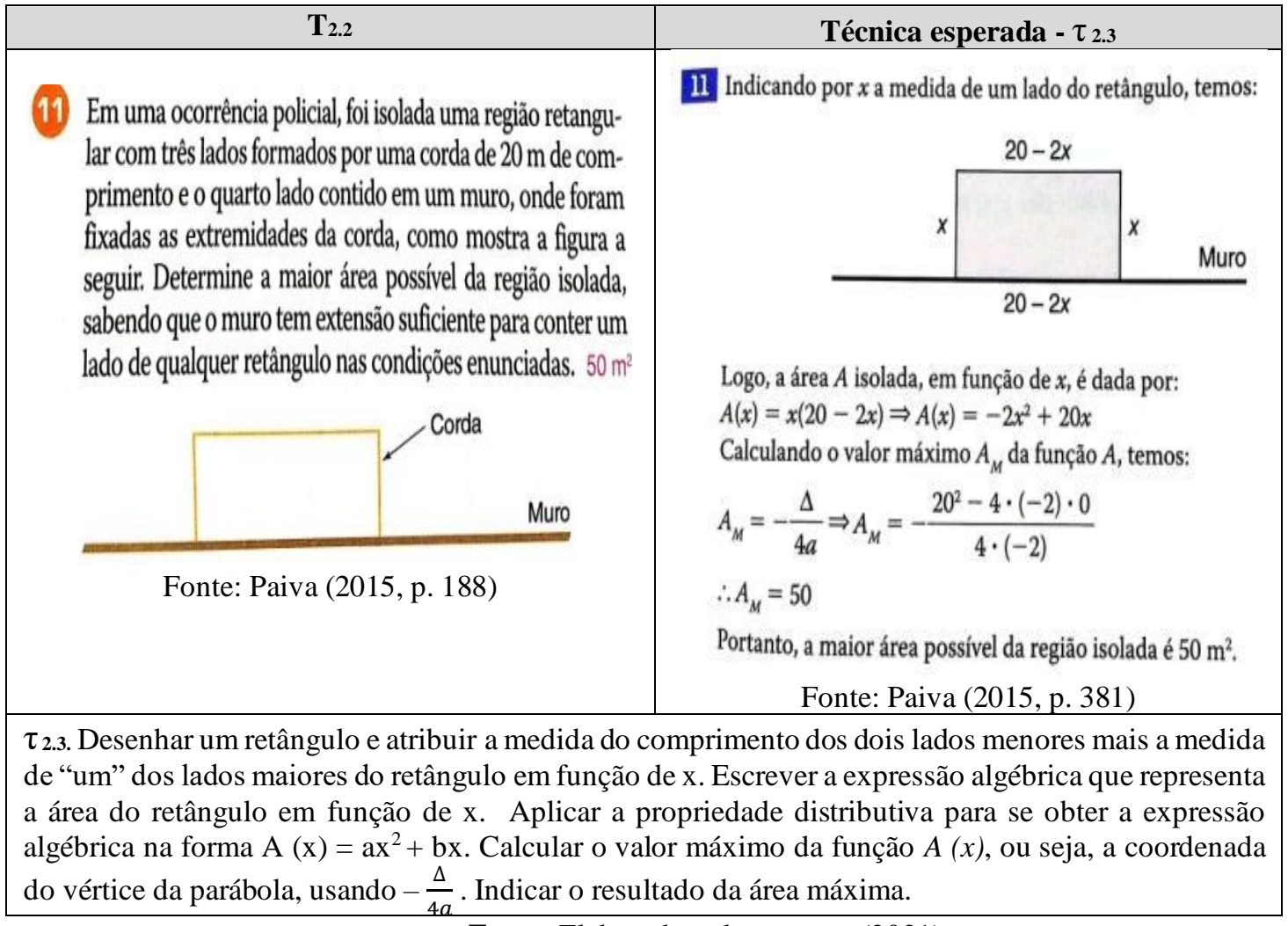

Fonte: Elaborado pelos autores (2021).

De modo geral identificamos quatro situações-problema relacionadas à T1, quatro relacionadas à T2 e apenas uma para T3. O LD1 e o LD3 foram aqueles que apresentaram a maior diversidade de tarefas.

Quadro 11 - Síntese dos resultados

\begin{tabular}{|c|c|c|c|c|c|}
\hline LD & Coleções & Tipc & e tarefas & Tipos & técnicas \\
\hline \multirow{5}{*}{ LD1 } & \multirow{5}{*}{$\begin{array}{l}\text { Matemática Ciência e Aplicações } \\
\text { (IEZZI et al., 2017) }\end{array}$} & subtipos & Frequência & Subtipos & Frequência \\
\hline & & $\mathrm{T}_{1.2}$ & 1 & $\tau_{1.2}$ & 1 \\
\hline & & $\mathrm{T}_{1.3}$ & 1 & $\tau_{1.3}$ & 1 \\
\hline & & $\mathrm{T}_{2.1}$ & 1 & $\tau_{2.1}$ & 1 \\
\hline & & $\mathrm{T}_{3.1}$ & 1 & $\tau_{3.1}$ & 1 \\
\hline LD2 & $\begin{array}{l}\text { Matemática Interação e Tecnologia } \\
\text { (BALESTRI, 2016) }\end{array}$ & $\mathrm{T}_{1.4}$ & 1 & $\tau_{1.4}$ & 1 \\
\hline \multirow{3}{*}{ LD3 } & \multirow{3}{*}{$\begin{array}{c}\text { \#Contato Matemática } \\
\text { (SOUZA; GARCIA, 2016) }\end{array}$} & $\mathrm{T}_{1.1}$ & 1 & $\tau_{1.1}$ & 1 \\
\hline & & $\mathrm{T}_{1.4}$ & 1 & $\mathrm{t}_{1.4}$ & 1 \\
\hline & & $\mathrm{t}_{2.1}$ & 1 & $\tau_{2.1}$ & 1 \\
\hline LD6 & $\begin{array}{l}\text { Conexões com a Matemática } \\
\text { (LEONARDO, 2016) }\end{array}$ & $\mathrm{T}_{2.1}$ & 2 & $\tau_{2.1}$ & 2 \\
\hline LD7 & $\begin{array}{l}\text { Matemática Ensino Médio } \\
\text { (SMOLE; DINIZ, 2014) }\end{array}$ & $\mathrm{T}_{1.1}$ & 1 & $\tau_{1.1}$ & 1 \\
\hline \multirow{3}{*}{ LD8 } & \multirow{3}{*}{$\begin{array}{l}\text { Matemática Paiva } \\
\text { (PAIVA, 2015) }\end{array}$} & $\mathrm{T}_{2.1}$ & 1 & $\tau_{2.2}$ & 1 \\
\hline & & $\mathrm{T}_{2.2}$ & 1 & $\tau_{2.3}$ & 1 \\
\hline & & Total T & 13 & Total t & 13 \\
\hline
\end{tabular}

Fonte: Elaborado pelos autores (2021). 


\title{
Conclusão
}

O objetivo deste trabalho em identificar a praxeologia matemática de situaçõesproblema envolvendo Função Quadrática e Área Máxima de Retângulo apresentadas em livros didáticos do Ensino Médio, traz à tona a relação intramatemática em situaçõesproblema sobre o tema Função Quadrática. Visto que:

\begin{abstract}
Deve-se observar que as articulações com as práticas sociais não são as únicas maneiras de se favorecer a atribuição de significados a conceitos e a procedimentos matemáticos, pois isso igualmente é possível, em muitos casos, com o estabelecimento de suas conexões com outros conceitos e procedimentos matemáticos importantes (BRASIL, 2006, p.95).
\end{abstract}

Vários autores, a exemplo de Barbosa (2004), reconhecem que a ideia de contextualização está, na maioria das vezes, associada apenas a conexões estabelecidas entre a Matemática e o cotidiano ou entre a Matemática e outras áreas do conhecimento. Dessa forma, esse autor, ao se referir à contextualização da Matemática, acrescenta:

\begin{abstract}
A utilização do termo "contextualização" tem sido indevida, já que todas as atividades da matemática escolar pertencem a um determinado contexto. Dessa forma, não cabe reivindicar a contextualização do ensino da Matemática. Ele já está contextualizado. A questão é outra. Qual é o contexto? Quais contextos desejamos (BARBOSA, 2004. p.2-3).
\end{abstract}

Grosso modo, constatamos sobre os oito LD analisados, que seis deles abordaram os tipos de tarefa T1, T2 e T3 (Quadro 11). E, utilizaram-se de contextualizações no cotidiano (semireais) e na própria Matemática. Dito isto, chamamos a atenção para a essência das tarefas e das técnicas em dadas situações-problema à luz da TAD, contextualizadas no cotidiano. Haja vista o exemplo da Figura 1 (uma área retangular para criar galinhas $-\mathrm{T}_{1.1}-\tau_{1.1}$ ) e o exemplo no Quadro 7 (uma área retangular para criar carneiros $\left.-\mathrm{T}_{1.4}-\tau_{1.4}\right)$.

Constatamos que o LD4 e o LD5 não apresentaram situações-problema no item “Atividades propostas", para os tipos de tarefas T1, T2 e T3, embora tenham apresentado exemplos desses tipos na sessão curso do capítulo de Função Quadrática (introdução e atividades resolvidas).

Ademais, esperamos que esse trabalho possa contribuir para outras pesquisas, voltadas ao tema Função Quadrática e Área Máxima de Retângulo, bem como, para outras formas de aplicação deste tipo de função. Sejam essas, a partir da análise de LD ou em outras perspectivas investigativas com a Teoria Antropológica do Didático. 


\section{Referências}

ARAÚJO, Abraão Juvêncio. O ensino de álgebra no Brasil e na França estudo sobre o ensino de equações do $\mathbf{1}^{\mathbf{0}}$ grau à luz da teoria antropológica do didático. 2009, 290 f. Tese (Doutorado em Educação) - Universidade Federal de Pernambuco, Recife, 2009.

BARBOSA, Jonei Cirqueira. A “contextualização" e a modelagem na educação matemática do ensino médio. In: ENCONTRO NACIONAL DE EDUCAÇÃO MATEMÁTICA, 8., 2004, Recife, Anais...Recife: SBEM, 2004.1 CD-ROM.

BALESTRI, Rodrigo. Matemática: interação e tecnologia. v.1. São Paulo: Leya, 2016.

BITTAR, Marilena. A Teoria Antropológica do Didático como ferramenta metodológica para análise de livros didáticos. Zetetiké, Campinas, SP, v.25, n. 3, set./dez.2017, p.364-387.

BRASIL. Orientações Curriculares para o Ensino Médio. Ciências da Natureza, Matemática e suas Tecnologias. Brasília: Ministério de Educação, 2006.

CHAACHOUA, Hamid, COMITI, Claude. L'analyse du role des manuels dans l'approche anthropologique. In : BRONNER, Alain et al. Diffuser les mathématiques (et les autres savoirs) comme outils de connassainces et d'action.Montpellier : IUFM, 2010, p. 771-789.

CHAVES, Adiel Praseres. Função Quadrática: análise em termos de contextos, de organizações matemáticas e didáticas propostas em Livros Didáticos de Ensino Médio. 2016. 111 f. Dissertação (Mestrado em Educação Matemática) - Faculdade de Ciências Exatas e Tecnologia, Pontifícia Universidade Católica de São Paulo, São Paulo, 2016.

CHAVANTE, Eduardo; PRESTES, Diego. Quadrante Matemática. v.1.São Paulo: SM, 2016.

CHEVALLARD, Yves. Concepts fondamentaux de la didactique: perspectives apportées par une approche anthropologique. Recherches en Didactique des Mathématiques, Grenoble,12(1), p.73-112, 1992.

DANTE, Luiz Roberto Dante. Matemática: contexto \& aplicações. v.1.São Paulo: Ática, 2017.

FLICK, Uwe. Introdução à pesquisa qualitativa. Trad. Joice Elias Costa. 3. ed., Porto Alegre: Artmed, 2009.

GARCIA, Jacqueline; SOUZA, Joamir. \# Contato matemática. v.1.São Paulo: FTD, 2016.

GODOY, Arilda Schmidt. Pesquisa qualitativa tipos fundamentais. Revista de Administração de Empresas, Rio Claro, v.35, n.3, mai./jun.1995, p. 20-29.

IEZZI, Gelson. et al. Matemática: ciência e aplicações.v.1. São Paulo: Saraiva, 2017. 
LEONARDO, Fabio Martins. Conexões com a matemática. v.1. São Paulo: Moderna, 2016.

PAIVA, Manoel. Matemática Paiva. Ensino Médio.v.1. São Paulo: Moderna, 2015.

SMOLE, Kátia Stocco; DINIZ, Maria Ignez. Matemática: Ensino Médio. v.1. São Paulo: Saraiva, 2014.

Recebido em: 28 / 02 / 2021

Aprovado em: 16 / 04 / 2021 